Document downloaded from:

http://hdl.handle.net/10251/39551

This paper must be cited as:

Triviño, A.; Ariza, A.; Casilari, E.; Cano Escribá, JC. (2013). On the Benefits of a Cooperative Layer-2 based Routing Approach for Hybrid Wireless Mesh Networks. China Communications. 10(8):88-99. doi:10.1109/CC.2013.6633748.

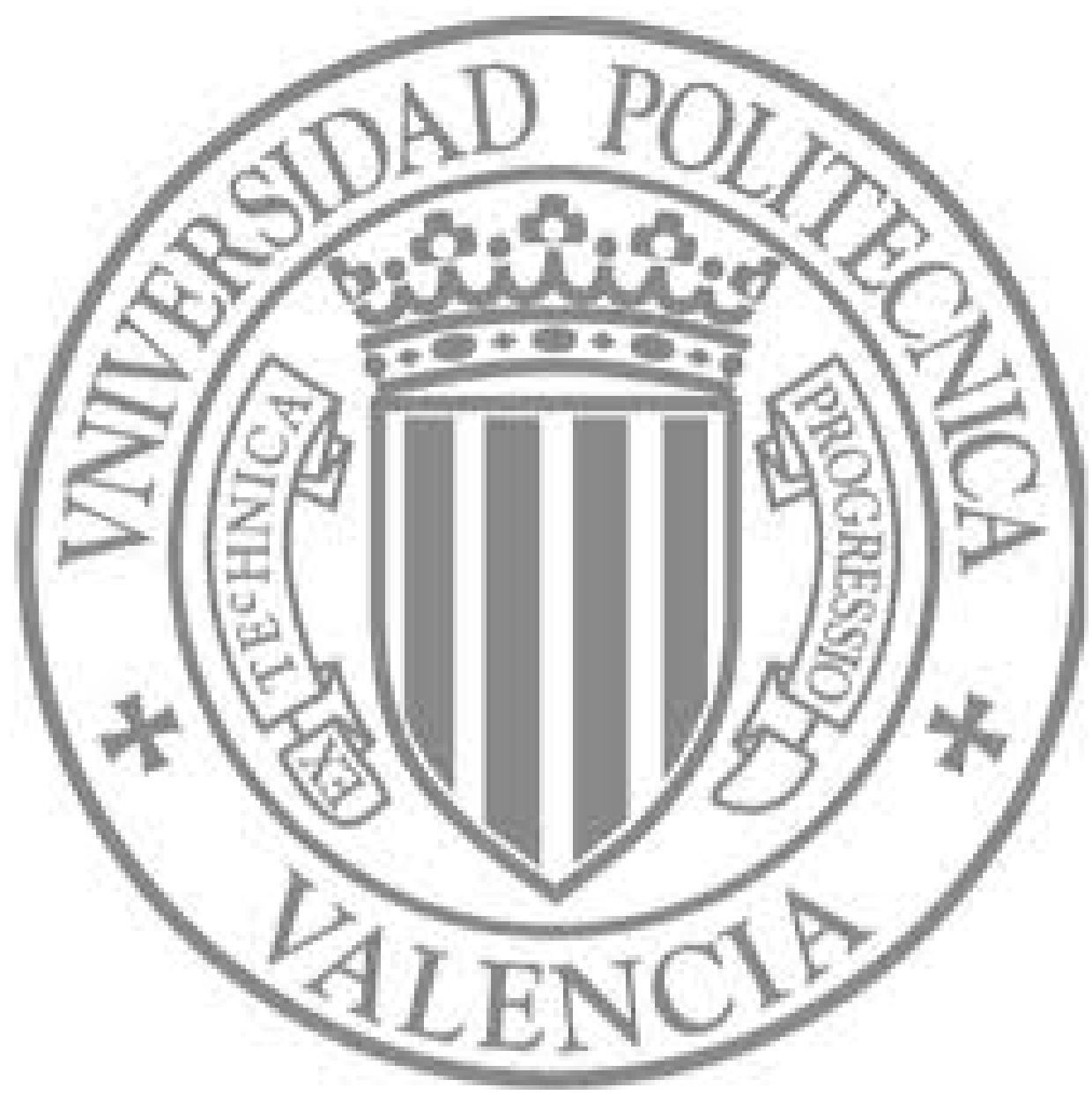

The final publication is available at

http://dx.doi.org/10.1109/CC.2013.6633748

Copyright Institute of Electrical and Electronics Engineers (IEEE) 


\title{
ON THE BENEFITS OF A COOPERATIVE LAYER-2 BASED ROUTING APPROACH FOR HYBRID WIRELESS MESH NETWORKS
}

\begin{abstract}
In a wireless mesh network, the convenience of a routing strategy strongly depends on the mobility of the intermediate nodes that compose the paths. Taking into account this behavior, this paper presents a routing scheme that works differently accordingly to the nodes mobility. In this sense, a proactive routing scheme is restricted to the backbone in order to promote the use of stable routes. On the other hand, the reactive protocol is used to search routes to or from a mobile destination. Both approaches are simultaneously implemented in the mesh nodes so that the routing protocols share routing information that optimize the network performance. Aiming at guaranteeing the IP compatibility, the combination of the two protocols in the core routers is carried out at the Medium Access Control (MAC) layer. Opposite to the operation at IP layer where two routing protocols are not able to concurrently work, the transfer of the routing tasks to the MAC layer enables the use of multiple independent forwarding tables. Simulation results show the goodness of the proposal in terms of packet losses and data delay.
\end{abstract}

Keywords: Hybrid Mesh networks, MANET, Hybrid routing protocols, layer-2 routing, layer-2 forwarding. 


\section{Introduction}

Nowadays, mobile devices such as PDA, phones or laptops are widely used. These popular devices are currently equipped with a wireless connection that allows users to get access to the Internet. When accessing to the Internet, several options are possible. Firstly, the devices could be connected by means of a cellular network (e.g. UMTS). However, this infrastructurebased technology requires the payment of the provided services. In order to avoid this cost, more economical solutions have been studied.

In this sense, community networks have prompted the development of WMN (Wireless mesh networks) in their urban centers [1] [2]. Taking advantage from the popularity and low-cost of IEEE 802.11 interfaces [3], a network of static wireless routers (backbone) is constructed. Some elements in the backbone, known as Gateways, can be connected to the Internet so that they provide access to any external hosts to the mesh nodes. A mobile device makes use of the wireless mesh network when it connects to one of the static routers (which will act as an Internet Access Router), from where the mobile node gets access to the Gateway, that is, to the Internet.

In order to communicate with the Gateway or any other mesh node, a multihop communication is established in the backbone. Thus, packets originated by the mobile devices are routed by the mesh nodes to guarantee that they reach the final destination. In some cases, the multihop communication is also extended for the mobile clients. These hybrid mesh networks may be contemplated as a particular case of a MANET (Mobile Ad Hoc Network) connected to a mesh network. In fact, some ad hoc routing protocols, which were conceived for MANETs, were initially tested for hybrid mesh networks. However, this kind of protocol needs to be optimized to benefit from the specific characteristics, i.e. the static positions and the power availability that mesh nodes have in comparison with the mobile ones. In this sense, specific routing protocols for wireless mesh networks have been proposed. As a result, the IEEE group has released the IEEE 802.11s standard [4] which supports wireless mesh networking. The nodes using the IEEE 802.11s executes the HWMP (Hybrid Wireless Mesh Protocol), which combines two complementary operating modes: a proactive scheme and a reactive one. The proactive procedure is exclusively used to maintain routes from any node to the Gateway and vice versa. Alternatively, the reactive scheme helps in searching routes between any two nodes. Consequently, routes to the Gateway are periodically computed and they are expected to be frequently used as most traffic is assumed to flow from or to the Gateway.

Since the routes to the Gateway, which may include one or several mobile nodes, are not always stable, the application of the IEEE $802.11 \mathrm{~s}$ routing strategy is not so beneficial in a hybrid WMN. In this paper, we will focus on optimizing the routing performance for this kind of topology by proposing a new routing protocol. Particularly, the main features of our routing proposal are:

(i) Promote the use of stable paths formed among the static routers. We propose to establish and periodically update the route to the Gateway but only in routes which just consist of static nodes. Since all these routes are stable and they usually offer a high capacity, it is recommended to keep all them updated in the static routers in order to prompt their use. Towards this goal, we suggest to deploy a proactive routing protocol in all the static routers. In contrast to [5] [6], the proactive scheme does not exclusively work to establish routes to/from the Gateway (as 802.11 is restricted to) but it also enables the discovery of routes between 
any two static routers. This additional knowledge that our proactive scheme offers could be exploited for the communications between two mobile nodes in the same mesh network. For instance, a mesh network which is built to support communications for a University campus will need to establish routing paths between the servers and the mobile clients, which could be attached to any static router.

(ii) Different routing policy according to the node's mobility. Although the mesh nodes are equipped with a proactive routing scheme, the mobile nodes are expected to provide a better performance when they execute a reactive protocol [7]. Thus, our proposal is supported by a proactive and a reactive routing protocol. The decision about which algorithm to use is based on the node's mobility.

(iii) Combination of a reactive and proactive routing scheme in the mesh nodes. Mesh routers are simultaneously provided with the reactive and the proactive routing protocols. The reactive routing protocol is necessary to supply the required bridge functionality so that the mobile nodes can access to the backbone.

(iv) Implementation at Layer-2. The routing procedures have been transferred to Layer 2 in order to enable a seamless use of their shelf Access Points. This transfer of the routing procedures is also justified in [8] [9] [10] [11]. Furthermore, the cross-layer design is extended to some other applications related to security issues [12].

To demonstrate the effectiveness and feasibility of our approach, we have implemented the algorithm in OMNeT++ simulation tool [13]. The simulation results show that the proposed scheme is able to increase the packet delivery ratio, reducing the packet delay while offering a high level of effectiveness.

The rest of this paper is structured as follows. Section 2 reviews the related work on hybrid routing protocols for wireless mesh networks. Section 3 details our proposed approach. The proposed routing approach is then evaluated using simulations in Section 4. Finally, Section 5 draws the main conclusions of this work.

\section{Related work}

The particularities of hybrid WMN and their effects on conventional MANET routing protocols was discussed in [14] [15]. As a result, the use of concurrent routing strategies for WMN has recently been proposed. The network nodes select the routing scheme to use depending on different conditions. In this sense, we distinguish the following criteria to activate the routing protocols:

A. Destination-dependent selection. In this group, the nodes execute one of the implemented routing protocols depending on the traffic destination node. For instance, AODV-ST (Spanning Tree) periodically updates the routes to the Gateway computed using a spanning tree [16]. Conversely, AODV [17] protocol is used to establish the communication paths between any other two nodes. A similar approach is followed by the standard IEEE 802.11s [4] and its routing protocol, that is, HWMP. The proactive procedure in HWMP is supported by a routing tree. In the routing tree, the root is the Gateway. This structure is periodically updated in the nodes, so the routes from any node to the Gateway and vice versa are continuously available in the nodes. On the other hand, the reactive scheme in HWMP helps for searching the routes between any other two nodes. The standard also contemplates the inclusion of RA-OLSR (Radio Aware - Optimized Link State Routing) as an alternative proactive routing protocol in 
HWMP [4]. RA-OLSR is a Layer-2 implementation of OLSR [18] (Optimized Link State Routing) which focuses on the quality of radio links to identify the best routes.

B. Mobility-dependent selection. In this group, the nodes execute a different routing algorithm if they are mobile or static. The work in [6] is one of the first proposals that differentiate the routing strategy according to this criterion. In a similar way to the previous schemes, it proposes to support the discovery of the routes from a Gateway to a static router by a proactive scheme. To do so, the Gateway periodically introduces a RREQ (Route Request) message to trigger the construction of a spanning tree. The main difference to other algorithms is that mobile nodes ignore this message. Therefore, only routes to the Gateway composed of static nodes are periodically computed. On the other hand, mobile nodes need to discover the route to the gateway using a reactive protocol when they need to communicate with external hosts. Similarly, [19] is intended for UAV (Unmanned Aerial Vehicles) applications so the mobility of the nodes in the network is heterogeneous. The aerial nodes implement a reactive protocol while the terrestrial ones select a proactive scheme. Alternatively, the proposal in [20] takes advantage from the resource availability in the static mesh nodes to promote the route maintenance activities in them. Specifically, when a route in use is detected to be broken, the border mesh nodes (the first static nodes in a path from the mobile node to the Gateway) are responsible for triggering the procedures to discover alternative routing paths. By this configuration, the wireless links are less occupied in comparison with a strategy where the final communication points initiate the route discovery. In addition, the mobile nodes do not consume their limited energy resources in performing these tasks.

Our cooperative scheme is mobility-dependent since the routing procedures differ in the mobile and in the static routers. Moreover, the proposed algorithm is also destinationdependent in the static routers. In fact, the static routers opt for a reactive or a proactive routing algorithm as a function of the mobility conditions of the destination. In contrast to the previous proposals, the selection of the routing policy in the mesh nodes does not only consider if the destination is a gateway or not but the mobility of the destinations. As a novelty, both routing schemes in the mesh routers interwork so that they can share some routing information and, in turn, improve the network performance.

\section{Cooperative Routing Protocol based on a Link Layer implementation for Hybrid WMNs}

In our proposed cooperative algorithm, mesh routers combine two routing protocols. The combination is done in order to enable and promote the exchange of routing information between the two routing structures. In particular, static routers are equipped with a proactive routing protocol and a reactive scheme. Alternatively, mobile nodes just implement the reactive ad hoc routing protocol. The election of this type of protocols in mobile nodes is justified by the study done in [7] where it is stated that reactive routing protocols perform better than proactive policies as mobility increases. In particular, we have selected AODVV2, also known as DYMO (Dynamic MANET On-Demand) [21], as the reactive protocol and OLSR (Optimized Link State Routing) [18] as the proactive scheme. By this selection, we aim at evaluating closely-related real WMN implementations as both protocols are being analyzed in the IETF (Internet Engineering Task Force) MANET working group for their standardization. In fact, AODVv2 and OLSR can be considered very representative candidates for the reactive and proactive families of ad hoc routing protocols. In addition, OLSR constitutes the basis for some commercial proposals for WMN such as the IEEE 802.11s [4] and BATMAN [22].

When a static router of the backbone needs to send a packet, the mesh nodes first look up a valid entry to the intended destination in the routing table built by the proactive algorithm. As only fixed nodes implement the proactive scheme, the selected paths will be exclusively 
composed of backbone nodes. This kind of path is usually more stable (failures because of the mobility of nodes do not occur) so traffic routed through mesh nodes does not experiment the degradation generated by route breakages.

On the other hand, a mobile node aiming at forwarding a message will search for a valid entry in its routing cache. If there is not any entry for the destination or if the route becomes invalid, the node will generate a RREQ (Route Request) message as it is specified in the conventional procedure of a reactive routing protocol. Mobile nodes receiving this message will retransmit the RREQ packet if they do not keep a route for the destination or they will respond with a RREP (Route Reply) message if they maintain a path to the destination. Conversely, the static nodes receiving a RREQ requires from the cooperation of their two routing protocols to process the message. Thus, they will firstly analyze if there is a valid entry in their proactive cache. In this case, the proactive protocol sends the original RREQ, with the data specifying the found route in the proactive tables, to its reactive protocol. The reactive scheme analyzes its own routing table. If both schemes (the proactive and the reactive) have found a valid route, the proactive path is selected because this route is considered more stable. On the other hand, when the proactive protocol does not keep a valid route to the demanded destination, the responsibility of discovering the path is completely transferred to the reactive protocol.

In order to implement both protocols without altering the conventional behavior of IP routing, it is necessary to transfer the routing tasks to Layer 2. In this sense, MAC layer is able to simultaneously work with several routing structures. At this level, packet headers contain four addresses associated to (i) the original source, (ii) the final destination, (iii) the next hop and (iv) the current relay node. The fields related to the next hop and the current relay nodes are updated at each and every hop. Specifically, the routing protocol is in charge of providing the MAC address of the next hop according to the information kept in the Layer-2 routing tables. An important benefit that can be derived from the Layer- 2 implementation is that the arrival of a packet can be used to directly update the routes to the source as the packet headers contain the necessary information for that purpose. The diagram in Figure 1 illustrates how these two protocols interact and how nodes can learn new routes when they retransmit a data frame. As can be observed, the proactive procedure looks up the routing information before the reactive protocol does it, when the two routing protocols are available in the static nodes. In this way, the information acquired by the proactive routing scheme has some priority with respect to the data obtained by the reactive policy. Consequently, the paths composed by static routers, that is, the paths discovered by the proactive routing protocol, are expected to be more frequently used than those containing mobile nodes. The paths connecting the static routers are expected to offer better quality as the losses due to the mobility of nodes are avoided [23].

Figure 1

Additionally, by implementing the routing procedures at Layer-2, it is possible to extend the coverage of the network adding new mesh nodes without consuming additional IP addresses as mesh routers will not require IP addresses.

Figure 2 illustrates how RREQ messages are propagated when a mobile node needs a route. In particular, Mobile node 2 aims at discovering the route to node $A$. Node $A$ is a static router so when this particular RREQ is firstly received by any static node, the route can be automatically constructed as the routes between the mesh nodes are periodically updated. We can confirm this behavior analyzing the sequence of messages. Firstly, Mobile node 2 sends a RREQ searching for Node A. The message is received by Mobile node 1 , which retransmits it because we assume that it has no routing information about this destination. Then, Node $B$ receives the 
RREQ. As it belongs to the backbone, it knows all the routes to all the static nodes. Therefore, it can directly reply to Mobile node 2 .

A different situation occurs when a mobile node searches for a route to another mobile node. In this example, this condition holds when mobile node 3 wants to communicate with mobile node 4 . If the nodes have not a valid route to node 4 in their reactive routing tables, the RREQ messages are propagated even by the static routers to the final destination in a reactive way. The dotted arrow represents the transmission of the RREQ message along the network. When received by the final destination (Mobile node 4), it replies with a RREP as illustrated by the blue arrow.

Figure 2

\section{Evaluation}

In order to evaluate the performance benefits of our approach, we compare it against other approaches under the same conditions (propagation, traffic, radio link rate, etc.). In particular, our study comprises a wide variety of traffic patterns and mobility conditions. We compare our cooperative approach against (i) a pure reactive routing protocol, (ii) a pure proactive protocol, (iii) a reactive protocol implemented at Layer 2, (iv) a proactive protocol implemented at Layer 2 , (v) a tree-based proactive protocol and (vi) a routing protocol proposed for hybrid WMN. In this way, the analysis we can do is threefold. Firstly, we can analyze the benefits of transferring the routing procedures to the link layer. Then, we can also study the advantages of combining different routing strategies (proactive and reactive) according to the destination. We also compare our proposal with a routing protocol intended for hybrid WMN [20]. Finally, under some circumstances, the comparison with a tree-based proactive protocol can be seen as evaluating the performance of HWMP against our proposal.

In our experiments, the simulation area is $2000 \times 2000 \mathrm{~m}^{2}$. In this area, there is a backbone composed of 49 mesh nodes equipped with wireless interfaces. The nodes are placed in a virtual grid as shown with circles in Figure 3. One of them is the Gateway, which is represented with a rhombus. The maximum transmission range is set to $250 \mathrm{~m}$ assuming a Free Space propagation model. Apart from this backbone, we have added 50 mobile devices. Every node moves at a constant speed to a destination point which is previously chosen. Once the destination is reached, the node searches for another point in the simulation area to which it will travel with a constant speed. The process is repeated until de simulation ends. This mobility pattern corresponds to a specific configuration of the well-known Random WayPoint mobility model [24]. Particularly, a non-null minimum speed has been set as recommended in [25]. In addition, setting a constant speed for the mobile nodes helps in obtaining scenarios with similar link durations at a given speed [26]. Thus, the confidence intervals, which inform about the statistical validity of the results, are reduced. Although this is not a realistic mobility pattern, we have selected it because it forces frequent link breakages and creations. In this sense, the simulated scenarios can be regarded as a worst case study.

Figure 3

Following our proposal, the static nodes are equipped with two protocols. In particular, they implement the proactive protocol OLSR (Optimized Link State Routing) [18] and the reactive scheme DYMO (Dynamic MANET On Demand) [21]. Conversely, mobile nodes just execute DYMO. The MAC layer protocol used in the simulation is the 802.11g [3] standard with a binary rate of $54 \mathrm{Mbits} / \mathrm{s}$. The rest of the simulation parameters are included in Table 1. 
Table 1

In order to quantify the network performance, we computed two metrics that inform about the quality perceived by network users. In particular, the following metrics are used:

- $\quad$ Packet Delay. It represents the mean time elapsed since a data packet is generated by a source until it arrives at the final destination. For those packets whose destination corresponds to a host in the Internet, we only compute the time necessary to reach the Internet Gateway so the effect of the external network (basically the Internet packet trip time) does not disturb our analysis.

- Packet Delivery Ratio. It is computed as the rate between the number of data packets that properly arrive at the intended destination node and the total number of data packets generated by the traffic sources. It corresponds to the inverse of the packet loss ratio.

To make the analysis realistic, the traffic sources may correspond to mobile or static nodes (Figure 3 shows the sources, which are marked by grey rectangles). By selecting a static router as traffic generator, we emulate the traffic concentration that occurs when several mobile nodes connects to a particular Access Point in a typical infrastructure way. Under these circumstances, Access Points publish the MAC addresses of the devices that are attached to it so other nodes can start communications with them. Additionally, some mobile nodes are also traffic generators. In fact, they are 10 sources in the static routers and 10 sources in the mobile nodes. However, the traffic injected by them differs.

Differentiating the traffic sources and the destination, we can evaluate how the routing schemes work when the probability of accessing from a mobile node increases. Particularly, two traffic patterns are studied:

- $\quad$ Pattern 1. In this group $80 \%$ of the traffic is generated by mobile sources.

- $\quad$ Pattern 2. It forces $80 \%$ of the traffic to be injected by static routers.

For both traffic patterns, communications are bidirectional. The destination for each communication is chosen randomly among the sources and the Gateway following a Uniform probability distribution function. The sources introduce 133.333 data packets/seconds into the network as UDP transfers. The size of the data packet is set to a constant value of 512 Bytes.

For each evaluated scenario, 5 runs with different seeds have been executed. The depicted figures represent the mean values of the measured metrics as well as the $95 \%$ confidence interval.

As a first step of our study, we evaluate the consequences of transferring the routing capabilities to Layer 2 . Thus, we compare the network performance of the following schemes:

- $\quad$ Reactive Protocol at Layer 2. In this case, the routing tasks of DYMO are transferred to Layer 2.

- $\quad$ Reactive Protocol at Layer 3. It represents the DYMO specifications performed at the IP layer.

- $\quad$ Proactive Protocol at Layer 2. It corresponds to the implementation of OLSR at Layer 2.

- $\quad$ Proactive Protocol at Layer 3. In particular, OLSR is used in this case. 
For traffic Pattern 1 and 2, Figure 4 shows the obtained packet delivery ratio. As can be observed, there are slight differences when the protocols are implemented at Layer 2 or Layer 3. This figure also shows that the packet delivery ratio is dramatically reduced for the proactive policies, especially when the node speeds increase. The information periodically acquired by the proactive protocol becomes obsolete in a short period of time under high mobility conditions. The learnt routes are useless to forward the packets and, consequently, the losses increase. This packet delivery ratio makes proactive protocols inadequate for common mobile applications.

Proactive protocols generate periodic routing messages so that the nodes can update their routing information. The availability of the routing information allows the transmission of the data frames without any delay. Conversely, they occupy the scarce wireless transmission resources and, consequently, they interfere with the data packets. As a result, proactive routing protocols lead to lower PDR results in comparison with reactive protocols. This behavior is also noticeable when the network becomes more dynamic, that is, the speed of the nodes increases. Under these circumstances, the routing information needs to be frequently refreshed with additional control messages. The higher the speed, more control packets are needed. We observe these phenomena in the figures.

Figure 4

Concerning both traffic patterns, when the source is mobile, its mobility tends to establish links with short lifetime in the communication path [23]. As a consequence, the routes used in the communications are unstable and losses are frequent. This effect is notable in traffic pattern 1, where a higher percentage of mobile sources exist. The packet delivery ratio shows how this traffic pattern is associated with a higher number of losses in comparison with traffic pattern 2.

As a conclusion, in this first step of our analysis we conclude that the impact of transferring the routing procedures to Layer- 2 is not dependent on the type of traffic access.

Due to the poor performance related to the proactive schemes, we exclude these two schemes (proactive at level 2 and proactive at level 3 ) in the representation of the following results. In this way, the results are much clearer to be interpreted.

We continue the comparative analysis studying our proposed cooperative approach (which is marked as "Hybrid" in the figures) against the following schemes:

- The reactive protocol implemented at Layer-2. In the previous analysis, we concluded that the reactive protocol leads to the best performance in terms of packet delivery ratio while offering a similar end-to-end delay.

- The tree-based routing protocol. It is based on the periodic construction of a tree routing protocol configured with the Gateway as the root. It corresponds to the implementation of the proactive scheme specified by the IEEE 802.11s standard. 
- The enhanced local repair algorithm. Proposed in [20], it transfers the route maintenance activities to the border mesh nodes.

Figure 5 and Figure 6 depict the packet delivery ratio and the packet delay for the four schemes respectively when the traffic pattern 1 characterizes the scenario. The proposed scheme leads to a reduced delay and a noteworthy improvement on the packet delivery ratio. These benefits are due to the simultaneous use of two routing schemes in the static routers. The routes established between the static routers are usually discovered by the proactive algorithm, and, consequently, they are immediately available when needed. Since the proposed algorithm promotes the use of the proactive routes, the packets can be forwarded without waiting for excessive route discovery procedures, as the rest of routing protocols require. In addition, the stable routes experiment less packet losses and, in turn, the packet delivery is improved. This benefit is not straightforward when a pure proactive routing policy is applied since the routing paths (periodically computed by the protocol) also contain mobile nodes. As a consequence, a pure proactive routing policy updates less stable routes than the proposed combination. The priority of the stable routes is gained because the information acquired by the proactive scheme is preferred when both routing protocols are executed in the same static nodes.

The inclusion of additional features in the static nodes outperforms a pure reactive scheme, as can be observed in the figures. The tree-based routing procedure also improves the network performance as the learnt routes (from the gateway to any node) may be used by one of the traffic sources.

Figure 5

Figure 6

Similar behaviors are detected when the network is evaluated under the traffic pattern 2 . Figures 7 and 8 represent the packet delivery ratio and the packet delay for these new traffic conditions. The main difference between both traffic patterns relies on the percentage of traffic generated by the static sources. In traffic pattern $2,80 \%$ of the traffic is due to the core nodes. These nodes firstly opt for the routes learnt by their proactive routing protocol. This preference is responsible for the increase in the packet delivery ratio. Although the used links are stable and they do not provoke excessive losses, the preference set in all the static nodes may lead to a congestion level. The congestion obliges packets to wait until in the nodes' queues until the links can be used. As a consequence, traffic pattern 2 experiments an increased packet delay.

Figure 7

Figure 8

In the previous experiments, a communication point could be the Gateway. By this configuration, the preceding simulations emulate most of the expected traffic pattern in a WMN where mobile user also access to the Internet. However, traffic could also be exchanged among mesh users if the WMN is developed for an institution. For instance, the users in a University can share some institutional documents (notes, forms, educational resources, etc) through the wireless mesh network. Thus, as a third part of our study, we analyze the network performance when the traffic is exclusively exchanged among the nodes (not flowing necessarily to/from the Gateway). The distribution of the traffic sources is equivalent to that of 
traffic pattern 1, i.e. $80 \%$ of the traffic is generated by mobile sources while $20 \%$ of the traffic is injected by the static routers. The sources introduce 133.333 data packets/seconds into the network as UDP transfers. The data packet length is 512 Bytes. Under these traffic conditions, IEEE 802.11s is supported by the reactive protocol implemented at Layer 2 . Therefore, our study compares the proposed algorithm to this type of protocol (marked as 'reactive' in the figures). Figures 9 and 10 show the results and they include the $95 \%$ confidence interval. In comparison with the reactive scheme, the packet delivery ratio clearly improves when the proposed schemed is used while the delay is also decremented. The other two reactive schemes (tree-based and enhanced local repair) obtain similar packet delivery ratio but the delay is considerable higher than the one experimented by the hybrid proposal. This behavior is motivated by the promotion of the routes formed by static mesh routers. These routes are stable so a lower number of losses occurs. In addition, they are periodically computed so that they are always available.

Figure 9

Figure 10

\section{Conclusions}

This paper proposes a cooperative routing scheme for hybrid wireless mesh networks, where a MANET can access to the WMN backbone. Our scheme combines two routing protocols and it is implemented at Layer 2 in order to guarantee the IP compatibility. The combination of these two routing protocols allows communication between nodes in the backbone to be supported with a proactive scheme while the communication with a mobile node can be executed with a reactive algorithm. By this combination, the use of any stable route (i.e. a path composed of static routers) is reinforced. When the proposed approach is used, the network performance is clearly improved in terms of delay and losses as shown by the simulation results. The experiments used for the evaluation comprise different conditions of mobility and traffic.

\section{References}

[1] AGUAYO D, BICKET J, BISWAS S, MORRIS R. RoofNet, experimental mesh network, http://pdos.csail.mit.edu/roofnet/doku.php

[2] freifunk, International Project for free wireless networks and frequencies http://start.freifunk.net/

[3] ABOUL-MAGD O. IEEE Standard 802.11 Overview. Wireless Local Area Networks Quality of Service: An Engineering Perspective [J]. Wiley-IEEE Press, 2008: 65 -96.

[4] IEEE Standard for Information Technology-Telecommunications and information exchange between systems-Local and metropolitan area networks-Specific requirements-Part 11: Wireless LAN Medium Access Control (MAC) and Physical Layer (PHY) specificationsAmendment 10: Mesh Networking, 2011.

[5] BAHR M. Proposed Routing for IEEE 802.11s WLAN Mesh Networks[C]//Proceedings of the $2^{\text {nd }}$ Int. Workshop on Wireless Internet (WICON). Boston, Massachusetts, United States, 2006.

[6] LE A, KUM D, CHO Y. An Efficient Hybrid Routing Approach for Hybrid Wireless Mesh Networks [J]. Lecture Notes in Computer Science, 2009, 5576: 532-542. 
[7] ARIZA A, TRIVINO-CABRERA A, CANO J C, CASILARI E, CALAFATE C, MANZONI P. Assessing the impact of link layer feedback mechanisms on MANET routing protocols[C]//Proceedings of the 14th IEEE Symposium on Computers and Communications (ISCC'09). Sousse, Tunisia, 2009, 770-775.

[8] GARROPPO R G, GIORDANO S, TAVANTI L. Experimental evaluation of two open source solutions for wireless mesh routing at layer two[C]//Proceedings of the International Conference on Wireless Pervasive Computing. Modena, Italy, 2010: 232-237.

[9] MAURINA S, FITZPATRICK J, TRIFAN L, MURPHY L. An Enhanced Bridged-Based Multi-hop Wireless Network Implementation[C]//Proceedings of the International Conference on Wireless Internet Conference (WICON). Singapore, 2010: 1-9.

[10] LODI A, MALAGUTI E, STIER-MOSES N E. Efficient and fair routing for mesh networks, Journal Mathematical Programming: Series A and B - Series B - Special Issue: Combinatorial Optimization and Integer Programming archive, 2010, 124 (1-2): 285-316.

[11] AVALLONE S, AKYILDIZ I F, VENTRE G. A Channel and Rate Assignment Algorithm and a Layer-2.5 Forwarding Paradigm for Multi-Radio Wireless Mesh Networks [J]. IEEE/ACM Transactions in Networking (TON), 2009, 17(1):267-280.

[12] HUI L, JIANFENG M, JIA H. SLCRM: Subjective Logic Based Cross Layer Reputation Mechanism for Wireless Mesh Networks [J]. China Communications, 2012, 9 (10): 40-48.

[13] VARGA A. OMNeT++ User Manual. http://www.omnetpp.org/

[14] KHAN S, PIRZADA A A, PORTMANN M. Performance Comparison of Reactive Routing Protocols for Hybrid Wireless Mesh Networks [C]//Proceedings of the $2^{\text {nd }}$ International Conference on Wireless Broadband and Ultra Wideband Communications (AusWireless). Sydney, Australia: 2007: 78.

[15] PIRZADA A A, WISHART R, PORTMANN M, INDULSKA J. ALARM: An Adaptive Load-Aware Routing Metric for Hybrid Wireless Mesh Networks[C]//Proceedings of the Australasian Conference on Computer Science. Wellington, New Zeland, 2009: pp. 37-46.

[16] RAMACHANDRAN K, BUDDHIKOT M, CHANDRANMENON G, MILLER S, BELDING-ROYER E, ALMEROTH K. On the design and implementation of infrastructure mesh networks $[C] / /$ Proceedings of IEEE Workshop on Wireless Mesh Networks (WiMesh). Santa Clara, California, USA: IEEE Press, 2005.

[17] PERKINS C, BELDING-ROYER E, DAS S. Ad hoc On-Demand Distance Vector (AODV) Routing. Request For Comments (RFC) no. 3561, July 2003.

[18] CLAUSEN T, JACQUET P. Optimized Link State Routing Protocol (OLSR). Request For Comments (RFC) no. 3626, October 2003.

[19] PEPPAS N, TURGUT D. A Hybrid Routing Protocol in Wireless Mesh Networks [C]//Proceedings of Military Communications Conference (MILCOM). Orlando, Florida, USA, 2007: 1-7.

[20] ALRAYES M, TRIPATHI R, TYAGI N, MISHRA A K. Enhancement of Route Maintenance in AODV over Hybrid Wireless Mesh Network[C]//Proceedings of the 1st International 
Conference on Recent Advances in Information Technology (RAIT). Dhanbad, India, 2012: 273278.

[21] CHAKERES I, PERKINS C. Dynamic MANET On-demand (AODVv2) Routing. IETF Internet Draft, October 2012 (Work in Progress). <draft-ietf-manet-dymo-23.txt>

[22] ANNESE S, CASETTI C, CHIASSERINI C F, DI MAIO N, GHITTINO A, REINERI M. Seamless Connectivity and Routing in Vehicular Networks with Infrastructure[J]. IEEE Journal on Selected Areas in Communications, 2011, 29 (3):501 - 514.

[23] TRIVIÑO CABRERA A, GARCíA DE LA NAVA J, CASILARI E, GONZÁLEZ CAÑETE F J, Application of Path Duration Study in Multihop Ad Hoc Networks [J]. Springer Journal of Telecommunication Systems, 2008, 38 (1-2): 2-9.

[24] CAMP T, NAVIDI W, BAUER N.“Improving the Accuracy of Random Waypoint Simulations Through Steady-State Initialization[C]//Proceedings of the $15^{\text {th }}$ International Conference on Modeling and Simulation. Marina Del Rey, California, USA, 2004: 319-326.

[25] YOON J, LIU M, NOBLE B. Random Waypoint considered harmful[C]//Proceedings of the Twenty-Second Annual Joint Conference of the IEEE Computer and Communications (INFOCOM), San Francisco, California, USA, 2003, 2: 1312 - 1321.

[26] CASILARI E, TRIVIÑO-CABRERA A. A Practical Study of the Random WayPoint Mobility Model in Simulations of Ad Hoc Networks[C]//Proceedings of the 19th International Teletraffic Congress (ITC19). Beijing, China, 2005: 115-124. 
Figure Captions:

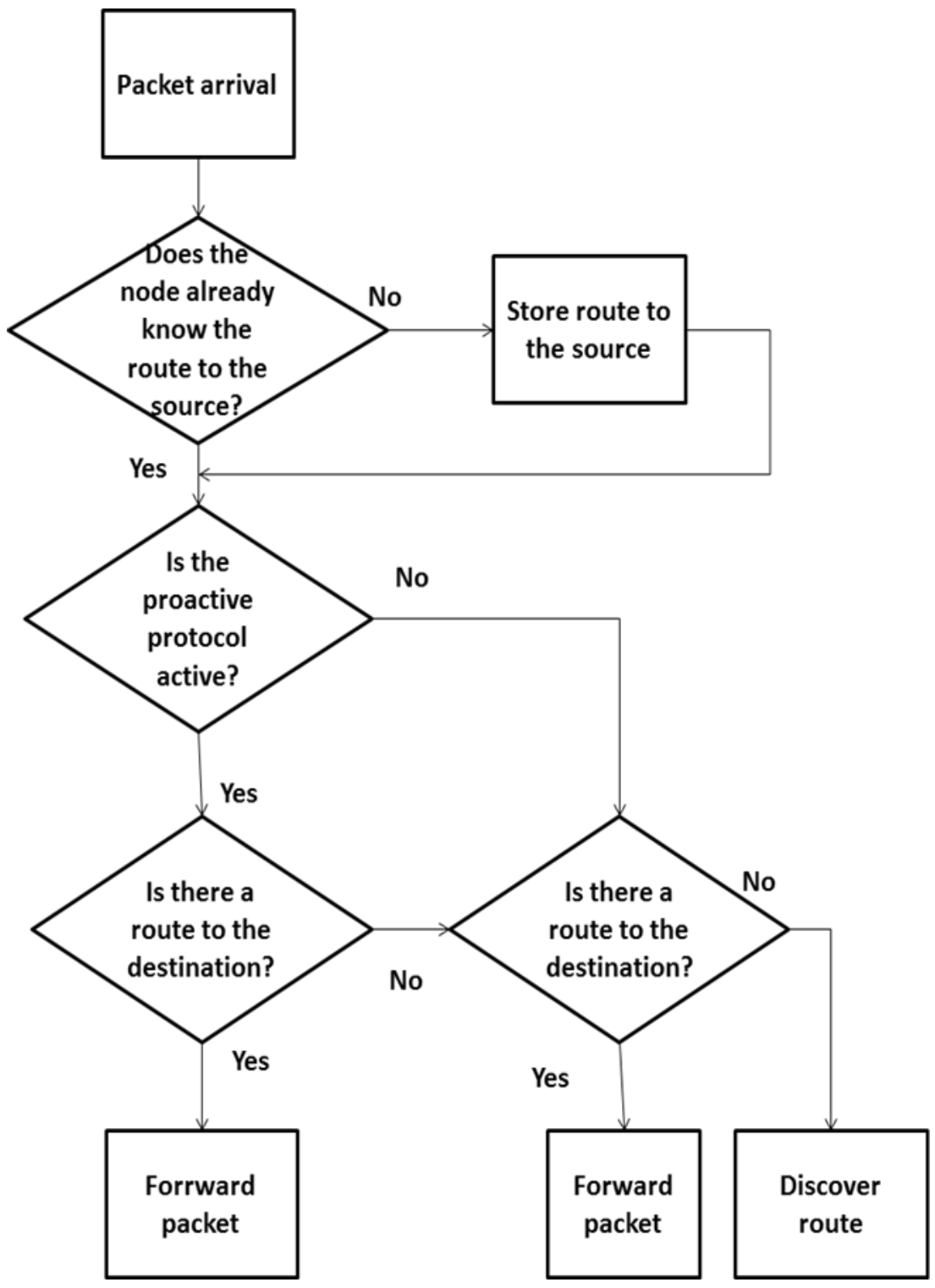

Figure 1. Packet forwarding mechanism in a static router. 


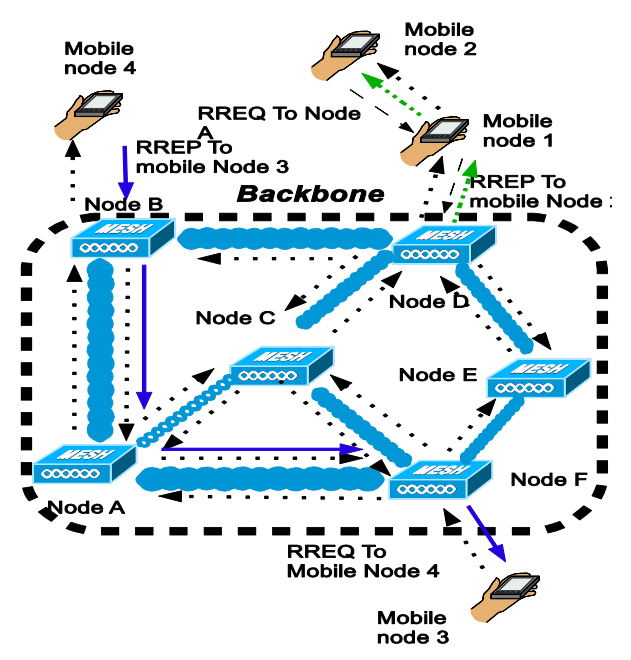

Figure 2. Illustrative example about how mobile nodes discover the routes in the mesh network.

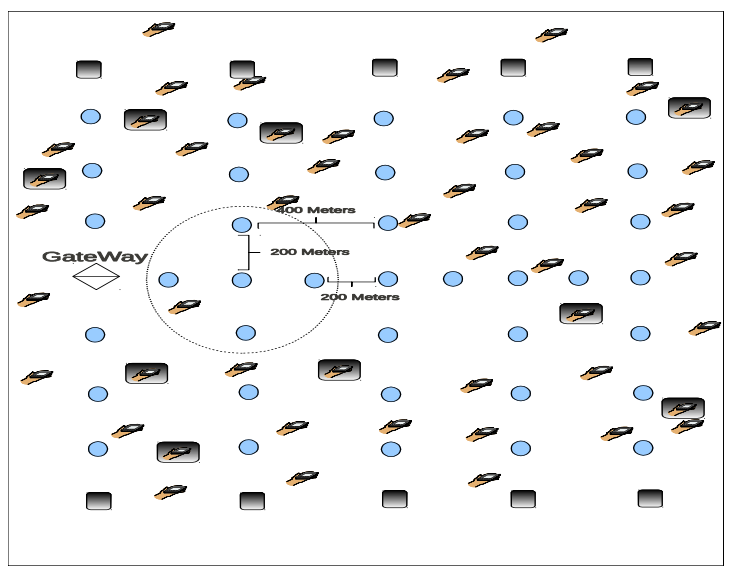

Figure 3. Structure of the evaluated mesh network. 


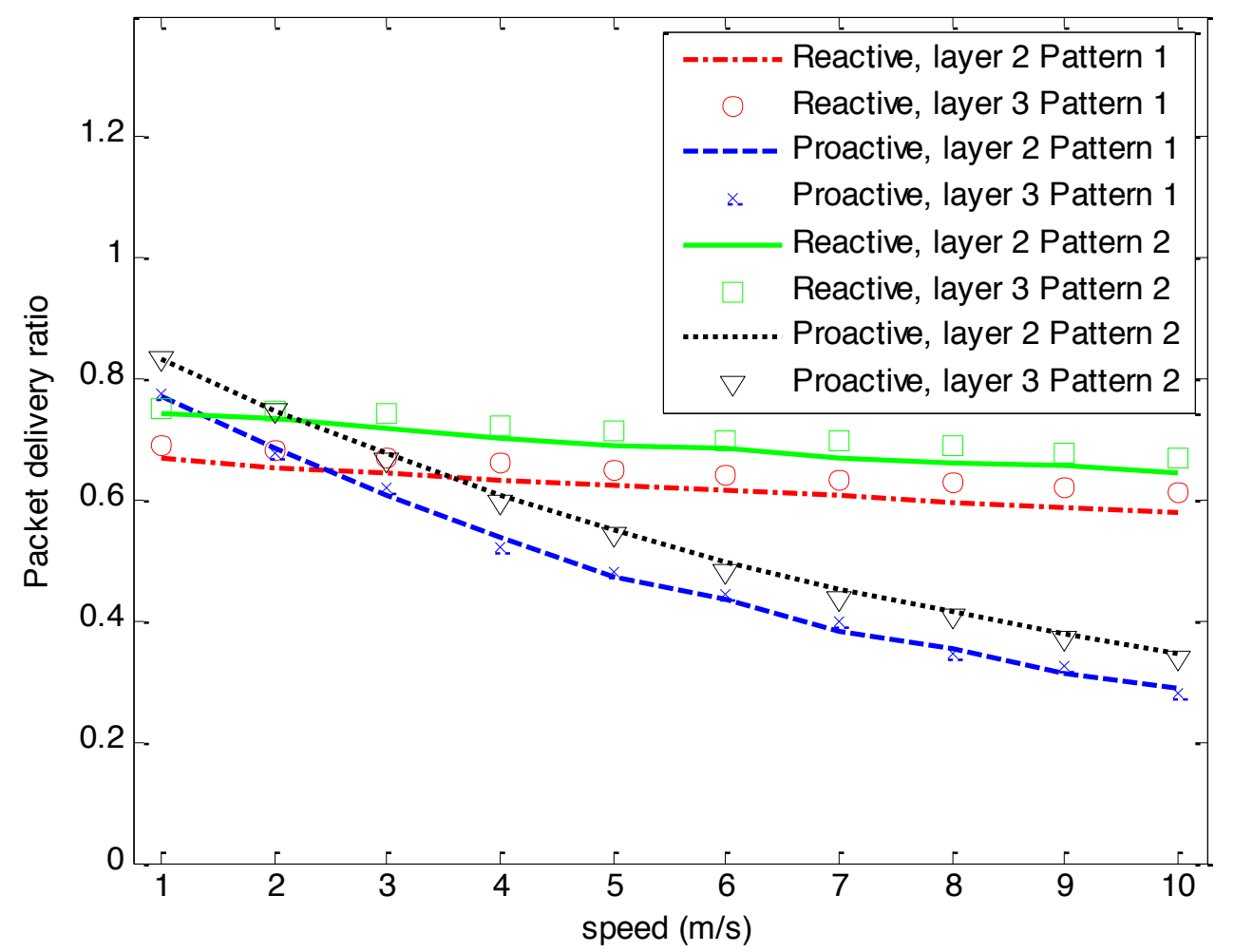

Figure 4. Packet delivery ratio for traffic pattern 1 and 2. 


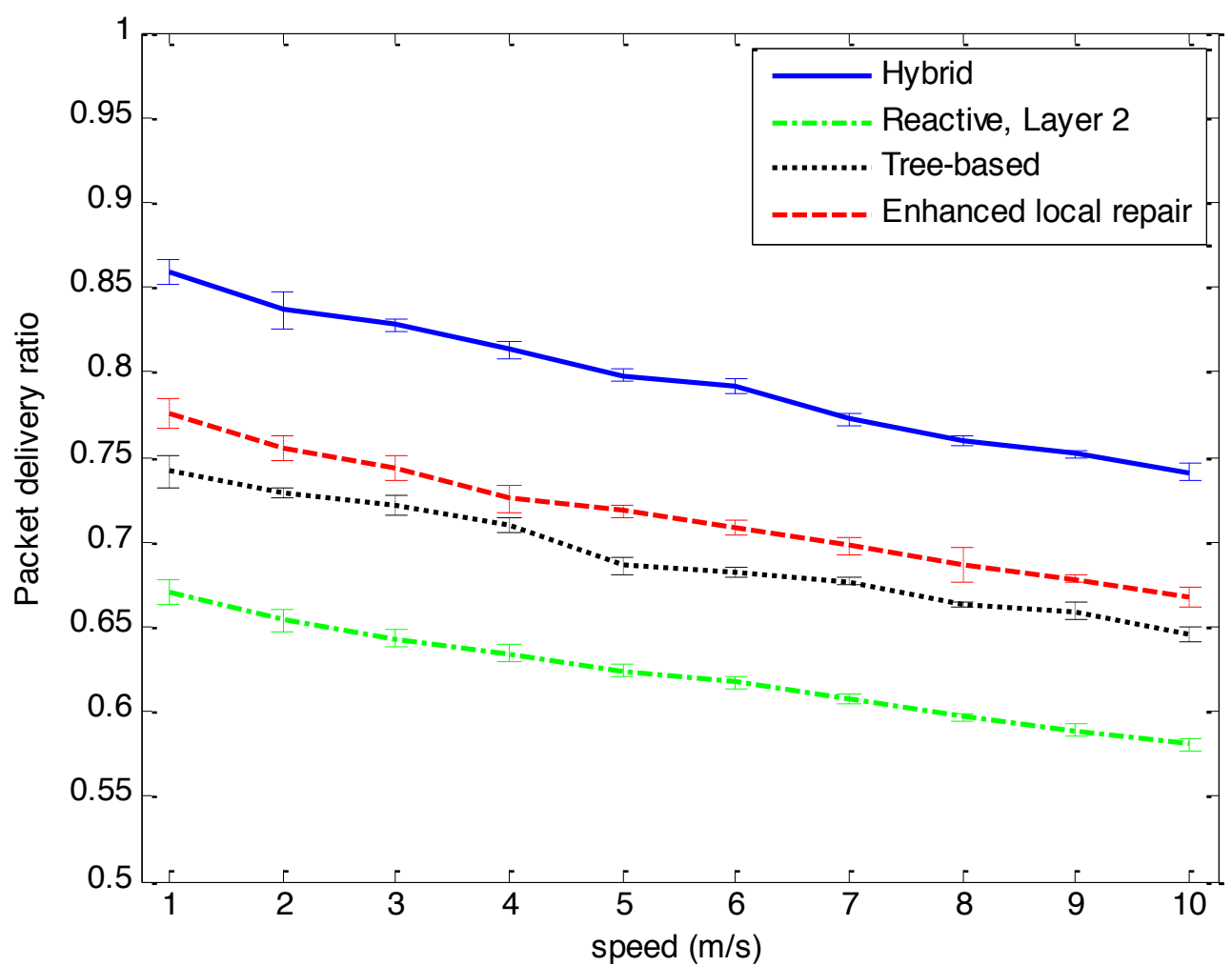

Figure 5. End to End Packet delivery ratio for Traffic pattern 1 (proposed hybrid protocol versus reactive routing, tree-based routing and routing with enhanced local repair) 


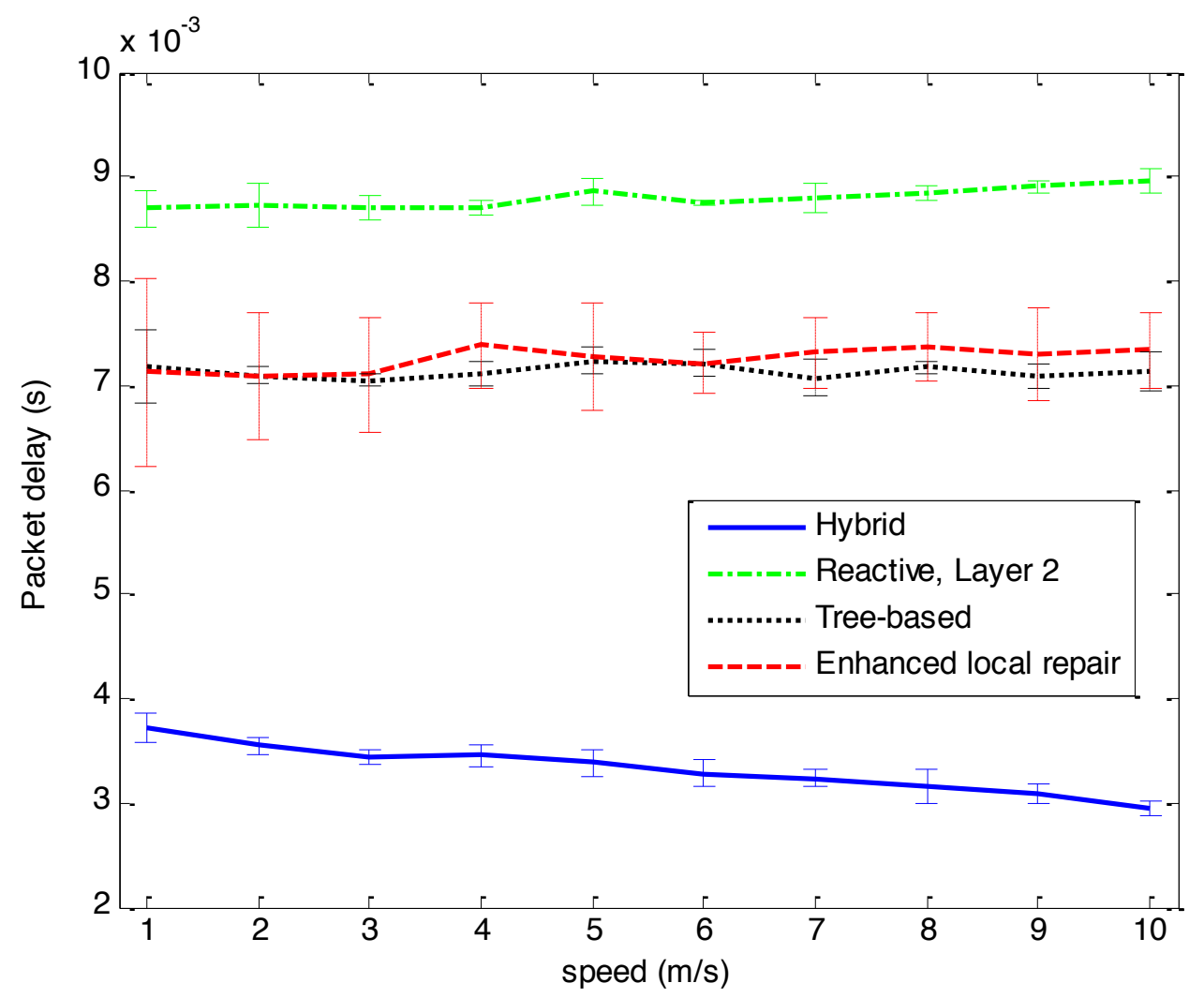

Figure 6. End to End delay for traffic pattern 1, proposed hybrid protocol versus, reactive and Proactive Tree 


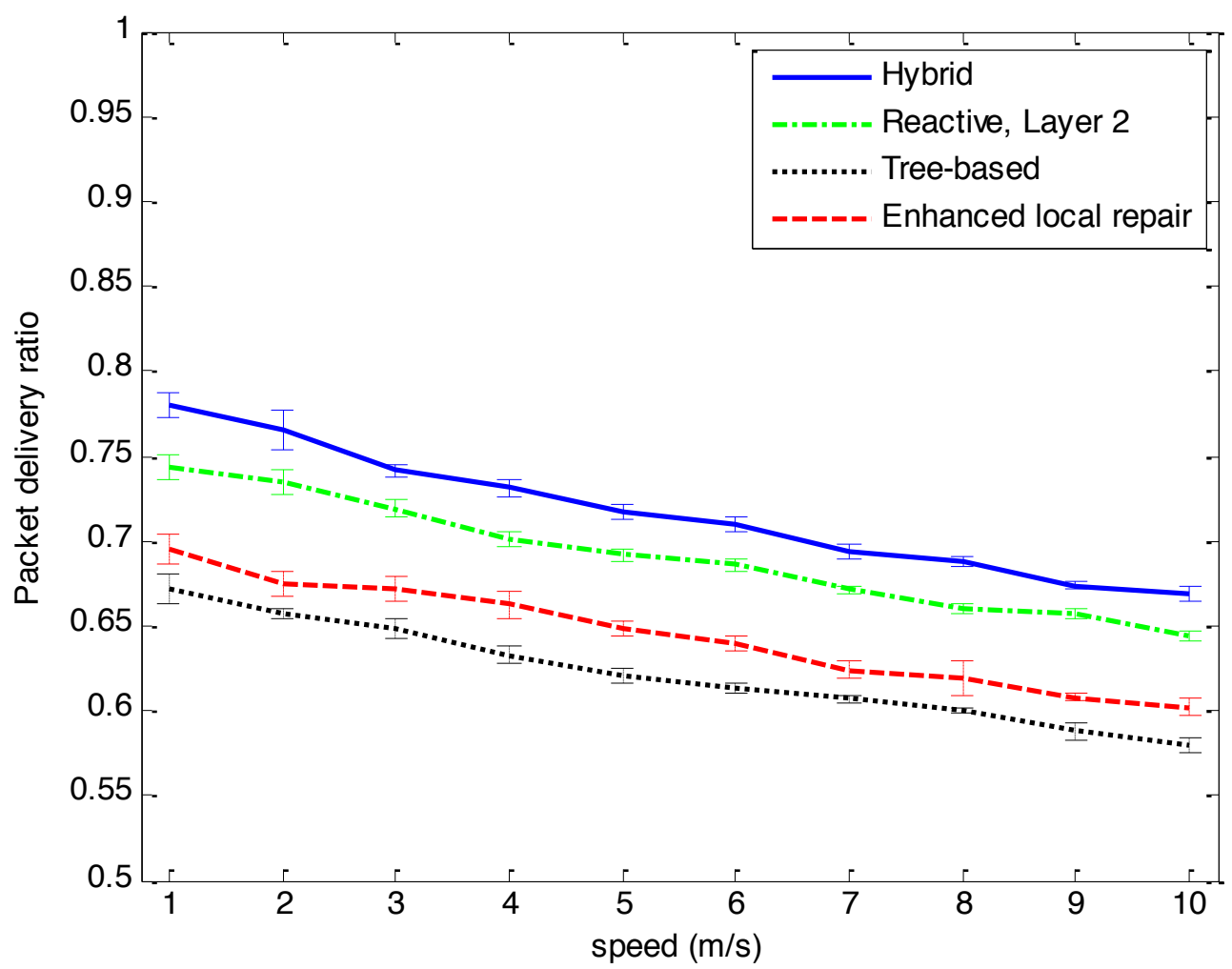

Figure 7. End to End Packet delivery ratio for traffic pattern 2 ( $80 \%$ traffic is generated by the static routers). 


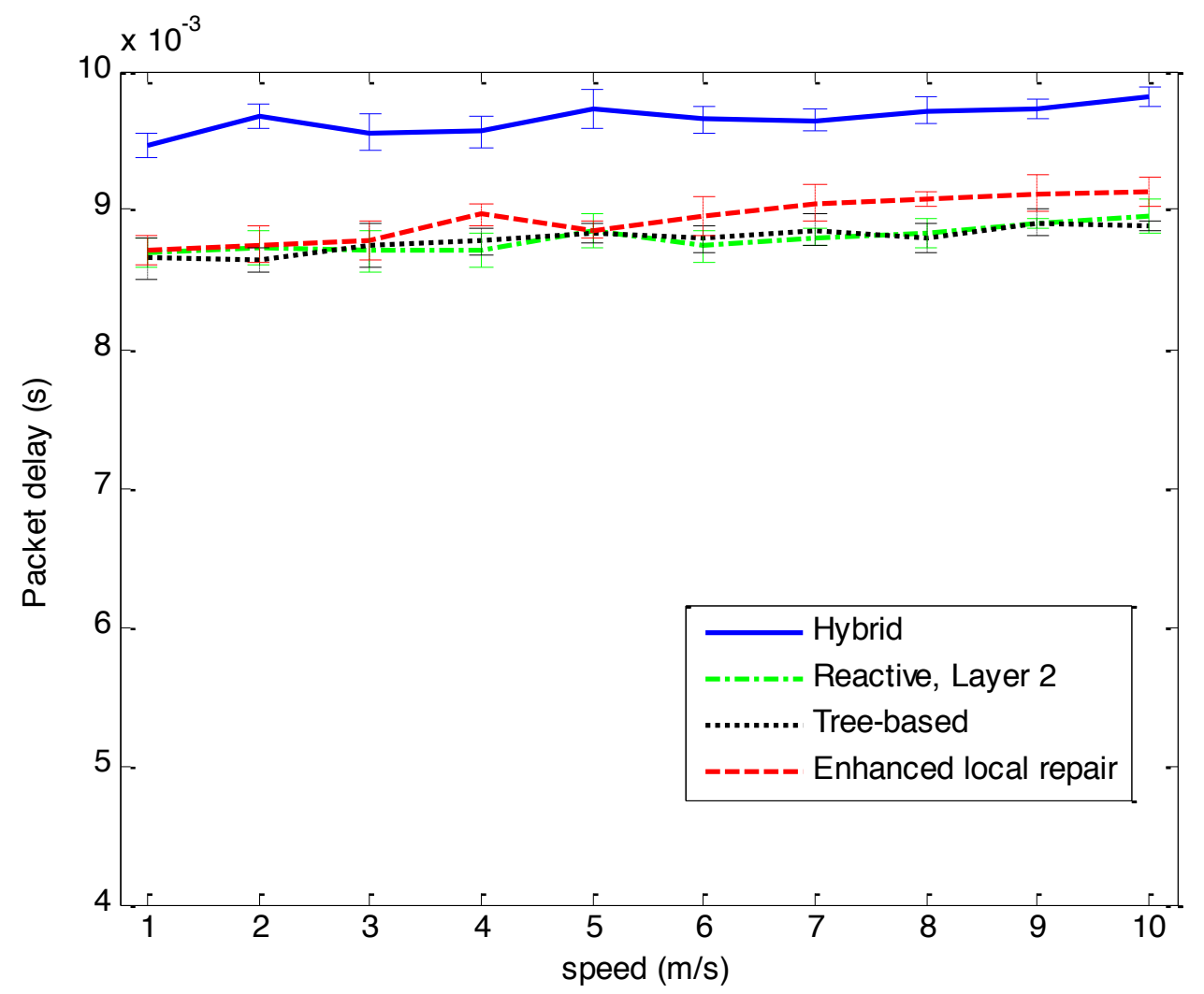

Figure 8. End to End delay for traffic pattern 2 ( $80 \%$ traffic is generated by the static routers).

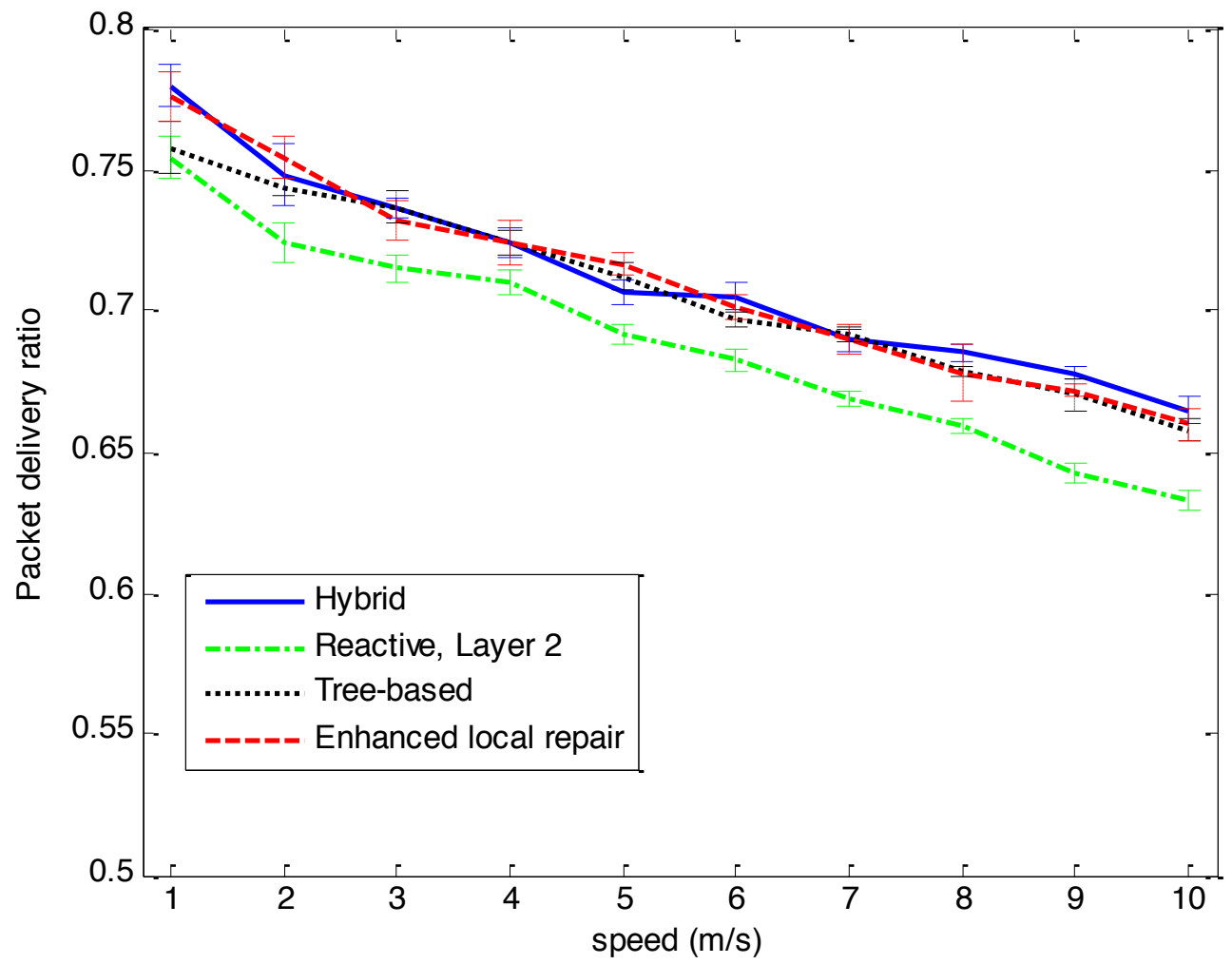

Figure 9. End to End Packet Delivery Ratio between any two nodes in the backbone. 


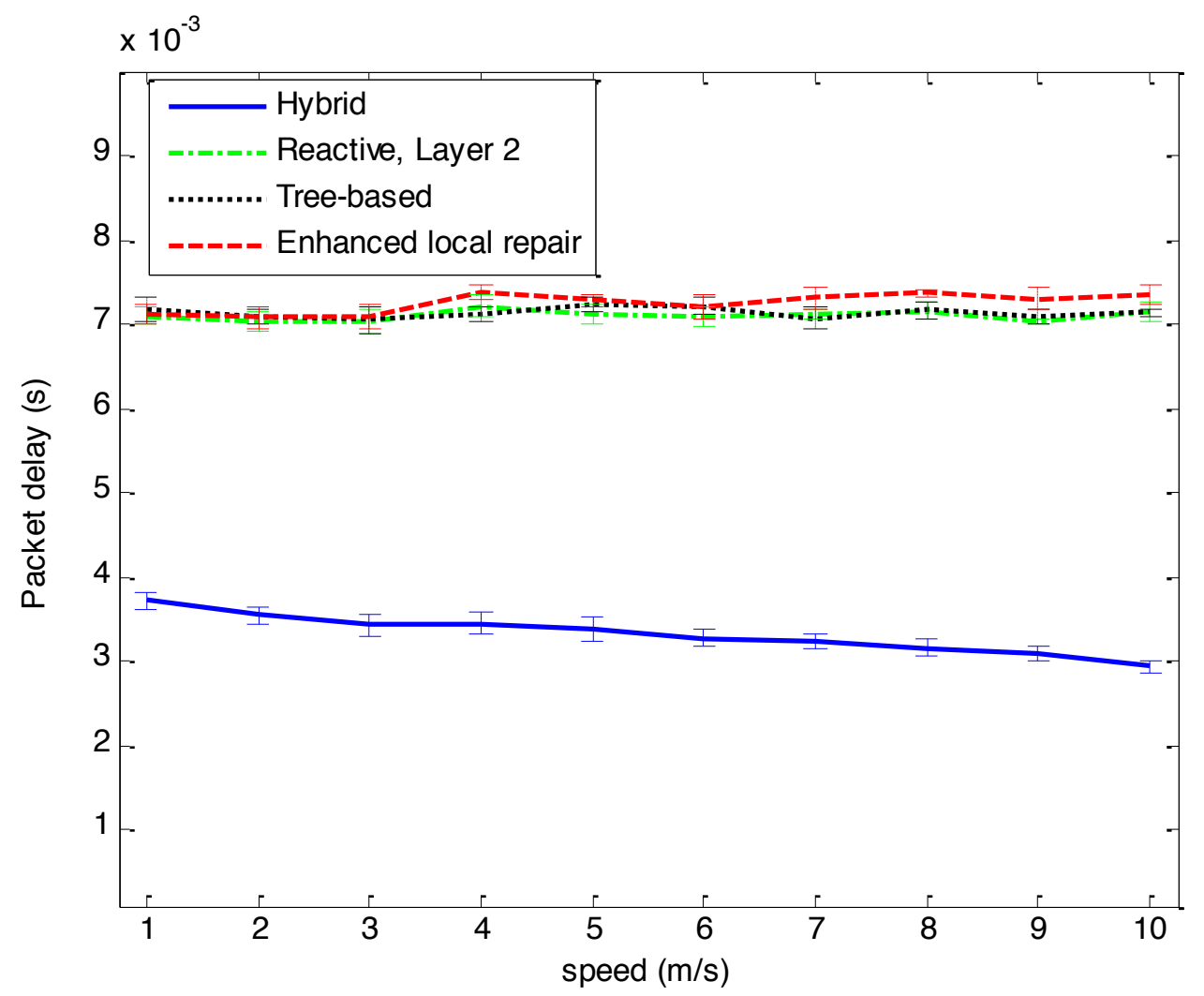

Figure 10. End to End delay between any two nodes in the backbone. 
Table 1. Simulation Parameters

\begin{tabular}{|c|c|}
\hline Simulation Area & $2000 \times 2000 \mathrm{~m}^{2}$ \\
\hline Number of mesh nodes & 49 \\
\hline Number of mobile nodes & 50 \\
\hline Mobile Nodes' Speed & {$[1,10] \mathrm{m} / \mathrm{s}$} \\
\hline $\begin{array}{l}\text { Pause Time } \\
\text { (after each displacement) }\end{array}$ & $0 \mathrm{~s}$ \\
\hline Propagation Model & Free Space \\
\hline Transmission Range & $250 \mathrm{~m}$ \\
\hline Interference Model & Additive \\
\hline MAC Layer & $802.11 \mathrm{~g}$ \\
\hline $\begin{array}{l}\text { Transmission Retry Limit (at } \\
\text { MAC Layer) }\end{array}$ & 7 \\
\hline Binary Transmission rate & $54 \mathrm{Mbits} / \mathrm{s}$ \\
\hline Hello Interval in OLSR & $2 \mathrm{~s}$ \\
\hline $\begin{array}{l}\text { TC (Topology Control) } \\
\text { Interval in OLSR }\end{array}$ & $5 \mathrm{~s}$ \\
\hline $\begin{array}{l}\text { Link Breakage Detection in } \\
\text { DYMO }\end{array}$ & Link Layer Feedback \\
\hline $\begin{array}{l}\text { Active Route Lifetime in } \\
\text { DYMO }\end{array}$ & $10 \mathrm{~s}$ \\
\hline $\begin{array}{l}\text { Additional routing in DYMO } \\
\text { RM packets }\end{array}$ & Yes \\
\hline Number of traffic sources & $\begin{array}{l}10 \text { sources selected from the mobile nodes } \\
10 \text { sources selected from the static routers }\end{array}$ \\
\hline Simulation time & $3000 \mathrm{~s}$ \\
\hline $\begin{array}{l}\text { Number of runs per } \\
\text { simulation }\end{array}$ & 5 \\
\hline
\end{tabular}

